\title{
"Metabolic endotoxemia" in the presence and absence of type 2 diabetes mellitus
}

\author{
Egshatyan L.V.1, Tkacheva O.N.1, Osipov G.A.2
}

1- National Research Center for Preventive Medicine, Moscow, Russian Federation

2- Zelinsky Institute of Organic Chemistry, Moscow, Russian Federation

\section{BACKGROUND:}

Type 2 diabetes mellitus (T2DM) is a condition of multifactorial origin, involving several molecular mechanisms related to the intestinal microbiota for its development. The microbiota able to favor systemic exposure to the lipopolysaccharides (LPS), large glycolipids derived from the outer membrane of Gram-negative bacteria. LPS can cause a condition of "metabolic endotoxemia" characterized by low-grade inflammation, insulin resistance.

\section{DESIGN:}

We performed a study in 21 individuals (10 patients with T2DM and 11 of controls without T2DM) mean age $56,12 \pm 13,2$ years and analyzed the relationship between levels of the lipopolysaccharides and body mass index, age, serum lipoproteins, fasting glucose, glycated hemoglobin $(\mathrm{HbA} 1 \mathrm{c})$ and the blood parameters of lowgrade inflammation.

\section{Criteria for exclusion from the study were as follows:}

Type 1 diabetes and other specific types of diabetes; regular intake of any drug (including antibiotics during the last 3 months, hypoglycemic drugs); severe diabetic microangiopathy (preproliferative and proliferative diabetic retinopathy, CKD 3b-5 stages); cardiovascular diseases: chronic heart failure class II-IV (NYHA), valvular heart disease; chronic liver and kidney failure; cancer; pregnancy; lactation; moderate and severe anaemia; infectious diseases; acute gastrointestinal tract diseases; the operations on the abdominal organs; diagnosed lactase intolerance; diagnosed allergic reaction to any food; a history of organ transplantation, diseases of the oral cavity and dentofacial system, refusal to participate in the study.
Study groups not differed significantly in terms of total serum cholesterol, low-density lipoprotein, high-density lipoprotein, triglyceride, interleukin-6, C-reactive protein, body mass index and age.

Group "without T2DM" and group "with T2DM" significantly differed in terms of $\mathrm{HbA} 1 \mathrm{c}$ level. Plasma LPS levels in T2DM patients were significantly different from those in controls (see table).

\begin{tabular}{|clll} 
& without T2DM & with T2DM & p \\
\hline HbA1c, $\%$ & $4,3 \% \pm 0,23 \%$ & $7,56 \% \pm 0,47 \%$ & 0,0005 \\
\hline LPS, nanomol/ml & $0,29 \pm 0,12$ & $0,46 \pm 0,31$ & 0,034 \\
\hline
\end{tabular}

Significant relationship between levels of the serum LPS and glucose $(r(s)=0,3 ; p=0,026)$, LPS and HbA1c $(r(s)=0,64$; $p=0,0049$ ) in all patients were revealed.

After separate into two groups, significant relationship between levels of the serum LPS and glucose $(r(s)=0,4$; $p=0,003)$, LPS and HbA1c $(r(s)=0,72 ; p=0,034)$ in T2DM patients were revealed.

In controls significant relationship were not revealed.

\section{Conclusion}

Patients with type 2 diabetes mellitus show higher lipopolysaccharides levels, which significant relationship with the serum levels of fasting glucose and $\mathrm{HbA} 1 \mathrm{c}$ unlike control group.

Due to the small number of patients in the group we didn't find a relationship between the levels of lipopolysaccharides and low-grade inflammation, and lipoproteins. 